\title{
A kind of Ball Rebound Tester for Flexible Cellular Polymeric Materials Nuo $\mathrm{Li}^{1, \text { a }}$, Tong Qin ${ }^{1,}$, Ming Yang ${ }^{1, \text { a }}$, Yue Wang ${ }^{1, \text { a }}$ \\ ${ }^{1}$ Electronic Institute, Liaoning Provincial Institute of Measurement of China, Wenhua road, Shenyang, 110004, China \\ aemail: nuoli@tju.edu.cn
}

\section{Keywords: Ball Rebound Tester; Calibration; Time Interval; Circuit Design; Rebound Value}

\begin{abstract}
The percentage rebound value $r$ is a key parameter for evaluating the resilience of flexible cellular polymeric materials. Usually, researchers use photoelectric detection method to construct the ball rebound tester. But the accuracy of ball rebound tester is hard to measure and keep. This paper analyzed the physics model by mathematical method, simplified the theoretical equation, compared two kinds of approaches mentioned in literature, developed a ball rebound tester, proposed and realized a calibration method for the tester in the end. Nowadays, the tester is distributed and the calibration method has been used in calibrating the ball rebound testers designed with the photoelectric detecting technique.
\end{abstract}

\section{Introduction}

Materials are characterized physically by their mechanical properties, which are measures of their response to external forces [1]. The percentage rebound value $\mathrm{R}$ is a key indicator which is used to reflect the resilience of flexible cellular polymeric materials [2]. The ball rebound tester is used to measure the percentage rebound value $\mathrm{R}$ for its fast and simple operation. At first, researchers use eyes to read out the rebound height. This method is inaccuracy, boring and exhausted. Then, some researchers adopt high speed camera to shoot the whole process. The cost of this method is higher. With the development of photoelectric detecting technique, many old ball rebound tester were replaced or improved by this technique.

Nowadays, researchers use photoelectric detection method to construct the ball rebound tester [1] [4]. Some researchers use two photoelectric detection sensors to measure the rebound height and some use one sensor. The circuit and logic control method are simple when using two sensors but need more cost. The measurement range is also reduced when using two sensors. For the two reasons above, many ball rebound testers adopt one sensor now. This paper will talk about the theory, principle design and calibration method.

In this paper, first we used differential equation to describe the physical process of a rebounding ball; second we analyzed the influence factors by numerical computation method, simplified the physical process and got the expression of rebound height; third we developed a kind of ball rebound tester; forth we calibrated our tester with oscilloscope and figure out a calibration method for nearly all kinds of ball rebound tester in the market with function arbitrary waveform generator.

This paper will be structured as follows; In Section II we will analyze the physics model by mathematical method; in section III we will simplify the theoretical equation and describe our approach and tips about system design; in section IV we will talk about the calibration methods for ball rebound tester; in the end, we will make a conclusion.

\section{Physics Model}

A steel ball is dropped on to a test piece from a specified height and the height of rebound is measured [5][6][7]. The force analysis of the ball is shown in Figure 1 and the physical process of a rebounding ball can be described as equation 1 according to Newton's second law of motion, where $g$ is the acceleration due to gravity, expressed in millimeters per second squared, $f_{\mathrm{d}}(t)$ is the air resistance and its direction is opposite to the ball, $m$ is the mass of the steel ball talked above, $a(t)$ is 
the accelerated velocity of ball, $z(t)$ is the position of the ball in z-axis. In this paper, the position of the ball means the position of ball's central of mass. We can get the equation 2, 3, 4 and 5 according to physical definition, where $v(t)$ is the velocity of ball, $C_{\mathrm{d}}$ is the aerodynamic resistance coefficient, $\rho$ is the air density, $A$ is the cross sectional area of ball and $R$ is the radius of ball. We can derive equation 6 from equation 1 to 5 .

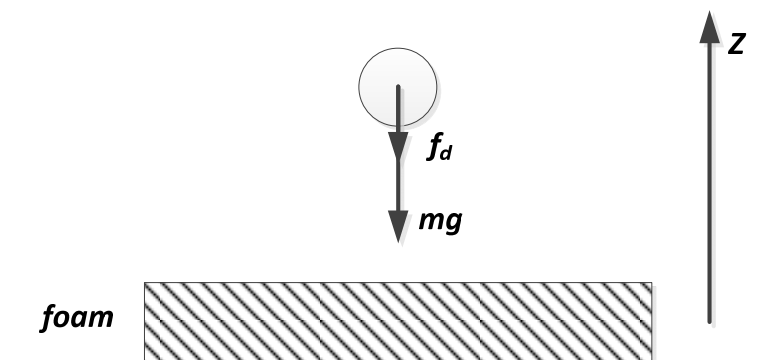

Fig.1. The force analysis of the ball

$$
\begin{aligned}
& m \times a(t)=m \times g+f_{d}(t) \\
& v(t)=\dot{z}(t) \\
& a(t)=\ddot{z}(t) \\
& f_{d}(t)=\frac{1}{2} C_{d} \times \rho \times A \times v^{2}(t) \\
& A=\pi \times R^{2} \\
& m \times \ddot{z}(t)=m \times g+\frac{1}{2} C_{d} \times \rho \times \pi \times R^{2} \times \dot{z}^{2}(t)
\end{aligned}
$$

The aerodynamic resistance coefficient $C_{\mathrm{d}}$ is a complicated parameter whose value is relevant to the shape and velocity of moving object. Asai T. researched the relationship between $C_{d}$ and Reynolds number, and creates table1 by a large number of experiments [8][9]. According to ISO 8307 method A, the height of drop is $500 \mathrm{~mm}$, the mass of the ball is $16.8 \mathrm{~g}$, the radius of the ball is $8 \mathrm{~mm}$ and $\rho$ is $1.293 \mathrm{~kg} / \mathrm{m}^{3}$. We can calculate the maximum velocity of the ball is about $3.1305 \mathrm{~m} / \mathrm{s}$ when it fell aboard of the test pieces ignoring air resistance and we can estimate the $C_{\mathrm{d}}$ is 0.5 according to table 1 . Then, the maximum $f_{\mathrm{d}}$ can be calculated by equation 4 and its value is 6.6553E-4 N.

Table1. The relationship between $C_{\mathrm{d}}$ and Reynolds number $R_{\mathrm{e}}$ (for footbal $)$
\begin{tabular}{|c|c|c|}
\hline \hline Velocity $(\mathrm{m} / \mathrm{s})$ & $R_{\mathrm{e}}\left(\times 10^{4}\right)$ & $C_{\mathrm{d}}$ \\
\hline 0 & 0 & 0 \\
2.5 & 3.63 & 0.5 \\
5.0 & 7.25 & 0.5 \\
7.5 & 10.9 & 0.5 \\
10.0 & 14.5 & 0.5 \\
12.5 & 18.1 & 0.5 \\
15.0 & 21.8 & 0.5 \\
17.5 & 25.4 & 0.5 \\
20.0 & 29.0 & 0.45 \\
$\ldots$ & $\ldots$ & $\ldots$ \\
\hline
\end{tabular}

\section{Model Simplification}

It's hard to find an analytical solution for equation 6 because it's a second-order nonlinear homogeneous differential equation. Usually, numerical method is used to solve equation 6 which needs a large amount of computation. During our research, we want to make a ball rebound tester which can measure the rebound height with a low-cost MCU automatically. Obviously, it's impractical to use numerical computation method on an ARM7 chip whose main frequency is 
72MHz. So we have to simplify the physics model.

It is easy to find that the value of maximum $f_{\mathrm{d}}$ is tiny. Can we ignore the air resistance? In this paper, we first adopt a four order Runge-Kutta method to find the influence of air resistance. We assume the velocity of ball is $3.1305 \mathrm{~m} / \mathrm{s}$ which is the maximum velocity when the rebound ball is just left the surface of test pieces. Then we can calculate the rebound curves by MATLAB. Figure 2(a) shows the rebound curve with air resistance, Figure 2(b) shows the rebound curve without air resistance, and Figure 2(c) shows the difference between the above two curves. It is easy to find that the air resistance has little effect on the ball rebound process.

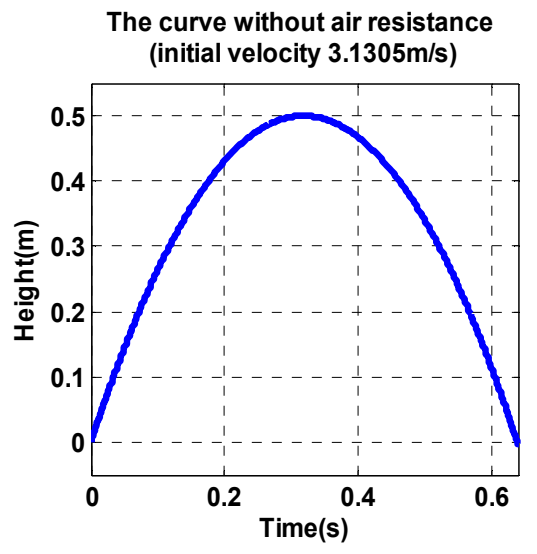

(a)

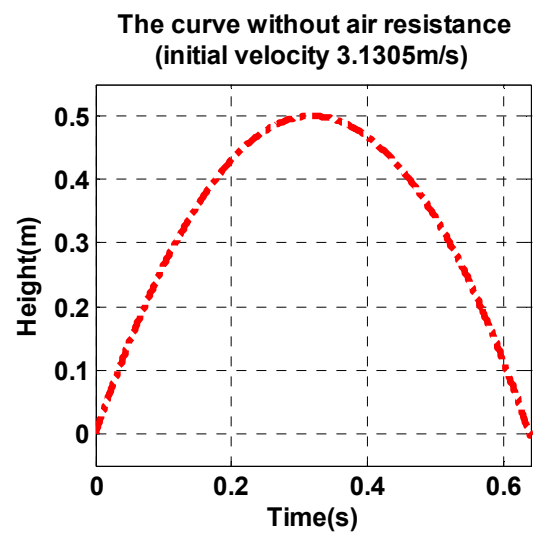

(b)

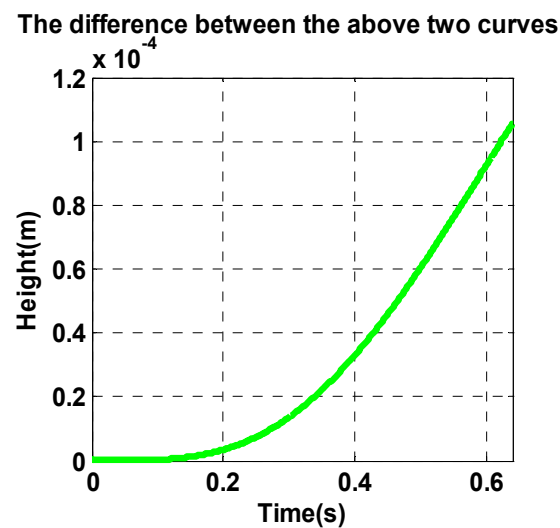

(c)

Fig.2. The influence of air resistance

Figure 2 is a qualitative evaluation. We can use the theorem of momentum to make a quantitative evaluation. Equation 7 is used to evaluate the effect of air resistance, equation 8 is used to evaluate the effect of gravity, where $M_{\mathrm{r}}$ is the momentum of air resistance, $M_{\mathrm{g}}$ is the momentum of gravity in the ball rebound process described above. We also assume the velocity of ball is $3.1305 \mathrm{~m} / \mathrm{s}$ when the rebound ball is just left the surface of test pieces and calculate the value of $M_{\mathrm{r}}$ is $1.3638 \mathrm{E}-4$ $\mathrm{kgm} / \mathrm{s}$, the value of $M_{\mathrm{g}}$ is $0.1054 \mathrm{kgm} / \mathrm{s}$. The effect of air resistance is nearly $1 \%$ of gravity which is much smaller than the design accuracy $1 \%$ of our tester. Then we can simplify equation 6 to equation 9 by neglecting the air resistance.

$$
\begin{aligned}
& M_{r}=\int f_{d}(t) d t=\int \frac{1}{2} C_{d} \times \rho \times A \times v^{2}(t) d t \\
& M_{g}=\int m g d t \\
& m \times \ddot{z}(t)=m \times g
\end{aligned}
$$

\section{Hardware Implementation}

Figure 3(a) shows the basic structure of our ball rebound tester which is completely in conformity with the clause in ISO 8307 [5]. The electromagnet is used to grip and release the steel ball which is controlled by a small control board placed in the host. The user can control the whole test procedure and read out the test result by operating the host. Figure 3(b) shows the motion curve of the ball, where $H_{1}$ is the free-fall height, $H_{2}$ is the center height of ultra-red photoelectric sensor device, $R$ is radius of the ball. Figure 3(c) shows the output of ultra-red photoelectric sensor device, where $t_{1}$ is the moment that the ball arrives at $H_{2}$ line when falling, $t_{2}$ is the moment that the ball all passes through $\mathrm{H}_{2}$ line when falling, $t_{3}$ is the moment that the ball arrives at $\mathrm{H}_{2}$ line when rebounding, $t_{4}$ is the moment that the ball all passes through $H_{2}$ line when rebounding, $t_{5}$ is the second time that the ball arrives at $H_{2}$ line when falling, $t_{6}$ is the second time that the ball all passes through $\mathrm{H}_{2}$ line when falling. According to ISO 8307, we need to calculate the rebound height $\mathrm{H}_{3}$ by measuring the time interval between $t_{3}$ and $t_{6}$.

Equation 9 is used to describe the physical process. We can construct the Equations 10, where 
$\Delta t$ is the time interval between $t_{3}$ and $t_{6}$ which can be measured by precision timing circuit. It is easy to find $H_{3}$ is at the moment just in the center of $t_{3}$ and $t_{6}$. We can use Equations 11 to calculate $\mathrm{H}_{3}$ which is the rebound height. We used STM32F103R as the MCU of our ball rebound tester, adopt its external trigger and timer function to record all the moment $t_{3}$ and $t_{6}$, and calculated the rebound value $r$ by equation 12 at last.
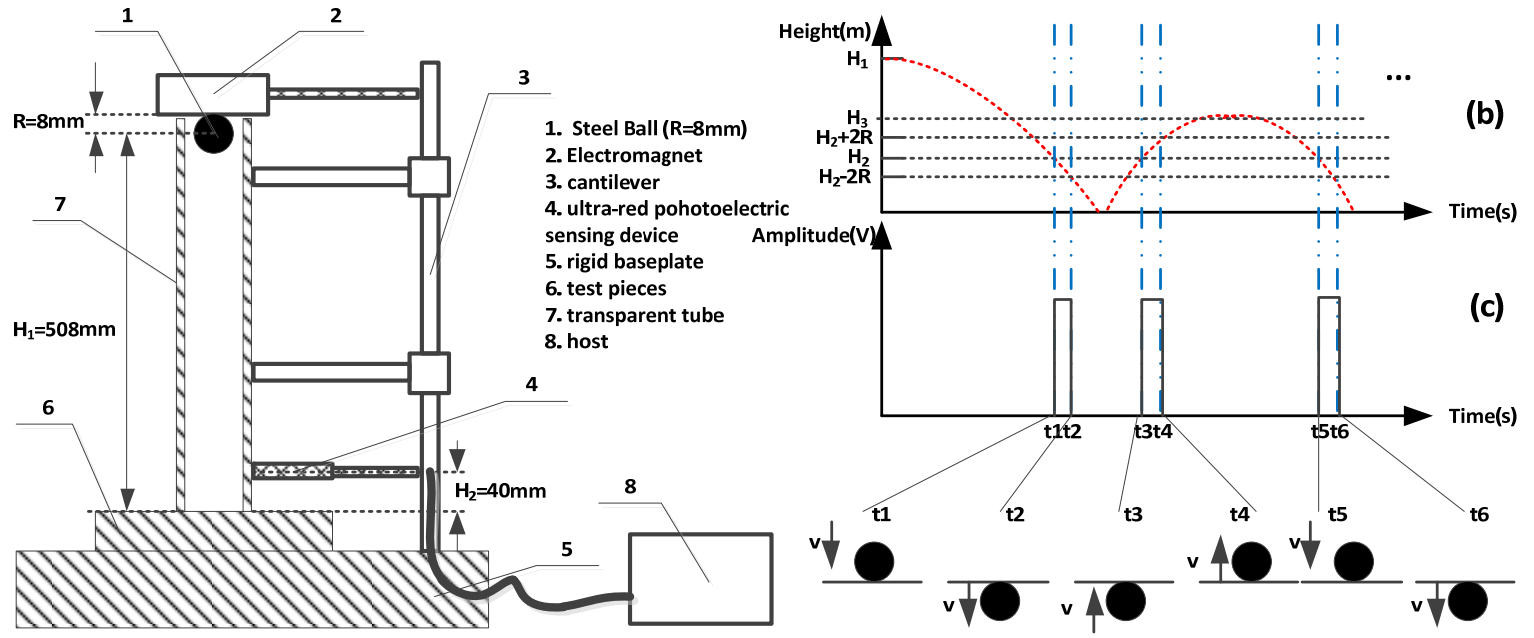

(a)

Fig.3. The basic structure of ball rebound tester

$$
\begin{aligned}
& \left\{\begin{array}{c}
m \times \ddot{z}(t)=m \times g \\
\ddot{z}\left(t_{3}\right)=\ddot{z}\left(t_{6}\right)=H_{2}-R \\
t_{6}-t_{3}=\Delta t
\end{array}\right. \\
& H_{3}=\frac{1}{8} \times g \times \Delta t^{2}+H_{2}-R \\
& r=\frac{H_{3}}{H_{1}} \times 100 \%
\end{aligned}
$$

\section{Calibration}

Li Xiangyang used high speed camera or video to calibrate the tester [10]. Although the method is easy to use but its accuracy is limited by the refresh rate of the camera or video. According to the normal refresh rate of video ( 25 frame per second), the maximum error is about $2 \mathrm{~mm}$. Besides, the mounting position of camera or video isn't horizontal because the rebound height is hard to estimate precisely. This can also import test error. In order to calibrate the tester, we use oscilloscope to record the output waveform of ultra-red photoelectric sensor and adopt the time cursors to measure the time interval $\Delta t$.

Usually, there are three connecting lines in ultra-red photoelectric sensor. One of them is GND line, one is power line, and the last one is the output line. We connect the output line and GND line of sensor to an oscilloscope (MSO4054) made by Agilent Company. Figure 4(a) shows our ball rebound tester and figure 4(b) shows the output waveform of the sensor. The first pulse (1) in figure $4(b)$ is the output waveform that the ball falls through the sensor; the second pulse (2) is the output waveform that the ball rebounds and passes through the sensor; the third pulse (3) is the output waveform that the ball falls through the sensor again; the forth pulse (4) is the output waveform that the ball rebounds and passes through the sensor again. The moment $t_{3}$ and $t_{6}$ are clearly seen by reference to Figure 3(c). It is convenient and accurate that calibrating the ball rebound tester by an oscilloscope for its time accuracy is about $2 \%$ when the screen span is $500 \mathrm{~ms}$. The test result is listed in table 2. According to the error computational formula we can get the precision of our system is about $1 \%$. 


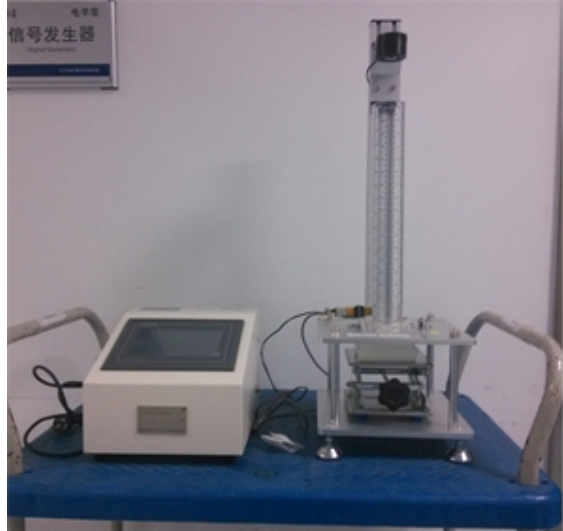

(a)

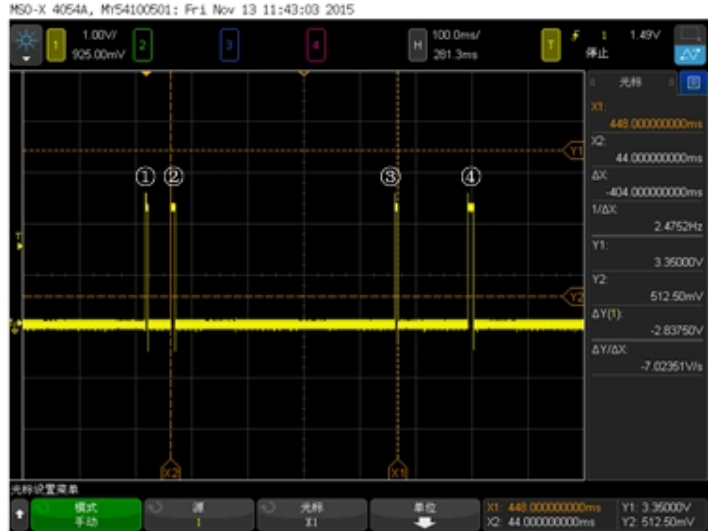

(b)

Fig.4. The ball rebound tester and its output waveform

Table2. Test result and error

\begin{tabular}{|c|c|c|c|}
\hline \hline $\begin{array}{c}\text { Time interval(ms) } \\
\Delta t\end{array}$ & $\begin{array}{c}\text { Theoretical } \\
\text { height }(\mathrm{mm}) H_{3}\end{array}$ & $\begin{array}{c}\text { Test result(mm) } \\
H_{3}\end{array}$ & Error(\%) \\
\hline 200.1 & 81.05 & 80.23 & -1.00 \\
302.7 & 144.24 & 143.17 & -0.74 \\
354.2 & 185.69 & 184.12 & -0.84 \\
404.5 & 232.43 & 231.84 & -0.25 \\
\hline
\end{tabular}

We can divide the calibration process of the tester into two steps in general. First, we need to check the function of the sensor. The step is a kind functional check. Figure 5(a) shows the connection diagram. Second, we need to check the measuring function of the host with a function/arbitrary waveform generator. The generator outputs a standard waveform to the host. If the amplitude of the waveform is not as high as the real sensor output, an amplifier should be used. The schematic diagram is shown in Figure 5(b). We can create a similar waveform like Figure 3(c) by the existing software (Agilent IntuiLink for Function/Arbitrary Waveform Generators) provided by Agilent company. For example, we use 10,000 points to make a waveform whose frequency is $1 \mathrm{~Hz}$. The time span of each point is $0.1 \mathrm{~ms}$. Then the time interval $\Delta t$ should be composed of $\Delta t / 0.1$ points. We can create a lot of precise test waveforms by following the rule. These standard waveforms can be stored in PC. The width of the pulse in figure 5(c) can be set as 10 points $(1 \mathrm{~ms})$. The time interval between pulse (1) and pulse (2) should be longer than 200 points $(20 \mathrm{~ms})$. The pulse (4) can be omitted.

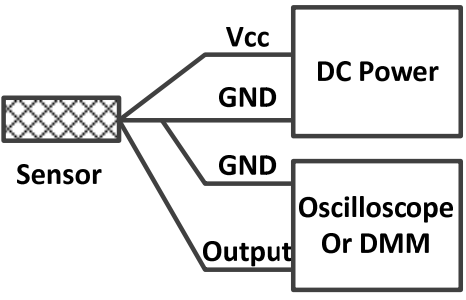

(a)

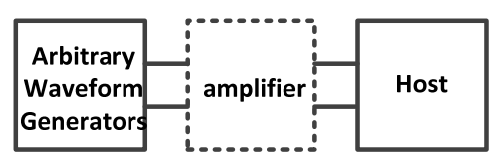

(b)

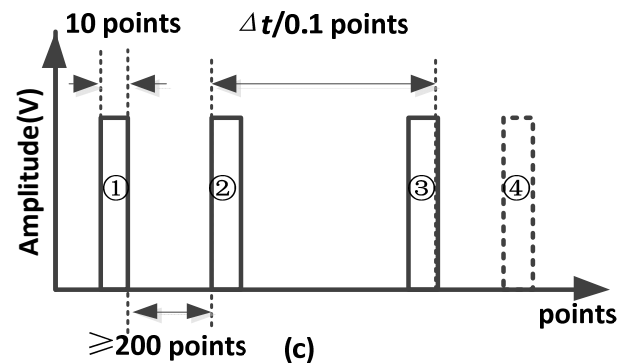

Fig.5. The calibration method for the ball rebound tester built by photoelectric detecting technique

\section{Conclusion}

Flexible cellular polymeric materials may have different requirements for resilience when it was applied in different environment or purpose. A ball rebound tester is used to evaluate the resilience of the foam. We built a kind of ball rebound tester based on photoelectric detecting technique and developed a kind of calibration method for the tester which based on the same technique also. We confirmed the accuracy of our tester is $1 \%$ by using the calibration method introduced in part V. 
It is easy to find that the accuracy and resolution of the tester depends on the measurement capability in time interval. The resolution of the timer in STM32F103R is not high enough. If we want to improve the accuracy and resolution of the tester, TDC-GP2 could be used which is a kind of special chip for measuring time interval. Whereas it is enough for the tester whose desired accuracy is $1 \%$.

The calibration method mentioned in part $\mathrm{V}$ has been used in calibrating the ball rebound tester. The same thinking can also be used in generating standard distorted signal, special time interval signal and vibration signal.

\section{Acknowledgement}

In this paper, the research was sponsored by the science and technology plan projects of AQSIQ (State Administration for Quality Supervision and Inspection and Quarantine) (Project No. T2016-LNQTS-0001) and science of public research fund of Liaoning Province (Project No. GY2016-A-003).

\section{References}

[1] T.Raphael, C.D.Armeniades. The "ADL Ball Rebound Tester" [J]. Polymer Engineering \& Science, 1964, 4(2): 83 89.

[2] Shi Hui. The development and application of rebound value tester for polyurethane foam [J]. Polyurethane Industry, 2002, 17(2): 48 50.

[3] Zhu Lifen. The ball rebound tester H-16[J]. Instrument Technique and Sensor, 1992, 1(1): 23 23.

[4] Zhang Jun. The application of soft time circuit for ball rebound tester [J]. Test Technology and Testing Machine, 1992, 32(1): 21 26.

[5] ISO 8307. Flexible cellular polymeric materials-Determination of resilience by ball rebound [S]. 2007.

[6] GB/T 6670. Flexible cellular polymeric materials-Determination of resilience by ball rebound [S]. 2008.

[7] ASTM D3574. Standard Test Methods for Flexible Cellular Materials_-Slab, Bonded, and Molded Urethane Foams [S]. 2003.

[8] Asai T, Akatsuka T and Haake S. The physics of football [J]. Phys. World, 1998, 11(6): 25 27.

[9] Yueqing Zhou, Zhaoning Ye and Zonghan Wu. The computation and analysis of aerodynamic resistance for ball games [J]. Physics and Engineering. 2002, 12(1): 55 59.

[10] Li Xiangyang. A discussion for measuring the accuracy of ball rebound tester HT-16 [J].Test Technology and Testing Machine, 1992, 32(1): 21 26. 